\title{
Pitavastatin reduces oxidative stress and attenuates intermittent hypoxia-induced left ventricular remodeling in lean mice
}

\author{
Sakiko Inamoto ${ }^{1}$, Toshitaka Yoshioka ${ }^{2}$, Chika Yamashita ${ }^{2}$, Masatoshi Miyamura ${ }^{1}$, Tatsuhiko Mori ${ }^{1}$, \\ Akira Ukimura $^{1}$, Chika Matsumoto ${ }^{2}$, Yasuo Matsumura ${ }^{2}$, Yasushi Kitaura ${ }^{1}$ and Tetsuya Hayashi ${ }^{1}$
}

We have reported previously that intermittent hypoxia related to sleep apnea induces cardiovascular remodeling secondary to the oxidative stress. The aim of this study was to examine the effect of pitavastatin as an antioxidant to prevent intermittent hypoxia-induced left ventricular (LV) remodeling in mice without hypercholesterolemia. Eight-week-old male C57BL/6J mice $(n=35)$ were exposed to intermittent hypoxia ( $30 \mathrm{~s}$ exposure to $5 \%$ oxygen, followed by $30 \mathrm{~s}$ exposure to $21 \%$ oxygen) for $8 \mathrm{~h}$ per day during the daytime or maintained under normoxic conditions; in addition, they were either treated with pitavastatin ( $3 \mathrm{mg} \mathrm{kg}^{-1}$ per day) or vehicle for 10 days. After cardiac catheterization and blood sampling, the LV myocardium was examined. The systemic blood pressure and plasma level of total cholesterol were similar among the four groups. Intermittent hypoxia significantly increased the expression levels of 4-hydroxy-2-nonenal (4-HNE) proteins, TNF- $\alpha$ and TGF- $\beta$ mRNA, and also the number of terminal deoxynucleotidyl transferase-mediated dUTP-biotin end labeling (TUNEL)-positive myocardial cells in the LV myocardium. In addition, enhanced hypertrophy of the cardiomyocytes, perivascular fibrosis and histological degeneration were observed in the mice exposed to hypoxic stress. Treatment with pitavastatin significantly suppressed the expression levels of the 4-HNE proteins, cytokines, superoxide production and TUNEL-positive myocardial cells in the LV myocardium, consequently attenuating the hypoxia-induced histological changes. Pitavastatin preserved, at least partially, the morphological structure of the LV myocardium in lean mice exposed to intermittent hypoxia, through its antioxidant effect.

Hypertension Research (2010) 33, 579-586; doi:10.1038/hr.2010.36; published online 19 March 2010

Keywords: HMG-CoA reductase inhibitor; hypoxia; oxidative stress; remodeling

\section{INTRODUCTION}

Sleep apnea syndrome has been known as an important risk factor for cardiovascular diseases such as systemic hypertension, pulmonary hypertension, congestive heart failure and cerebrovascular events. ${ }^{1-3}$ Several mechanisms, including inflammation, endothelial dysfunction and increased production of reactive oxygen species have been suggested to be involved in the development of cardiovascular dysfunction in these patients.

Oxidative stress has been known to have an important function in the progression of left ventricular (LV) remodeling. ${ }^{4}$ Recently, we reported intermittent hypoxic stress induces cardiomyocyte hypertrophy and interstitial fibrosis, and also increases superoxide production in the LV myocardium. These findings are not observed in mice with knockout of gp91phox, a constituent of NADPH oxidase, which is a major source of reactive oxygen species. ${ }^{5}$ Taken together, oxidative stress might be an important target to prevent $\mathrm{LV}$ remodeling caused by intermittent hypoxia.

The HMG-CoA (3-hydroxy-3-methylglutaryl-coenzyme A) reductase inhibitors or statins have been reported to have pleiotropic effects such as improving endothelial function, ${ }^{6}$ enhancing the stability of atherosclerotic plaques, ${ }^{7}$ accelerating ischemia-induced angiogenesis, ${ }^{8}$ decreasing oxidative stress, ${ }^{6}$ suppressing inflammation ${ }^{9}$ and inhibiting the thrombogenic response. ${ }^{10}$ Furthermore, statins have been reported to attenuate LV hypertrophy, ${ }^{11,12}$ and Girgis et al. ${ }^{13}$ showed that simvastatin attenuates chronic hypoxia-induced pulmonary hypertension in rats. Thus, pitavastatin, one of the HMG-CoA reductase inhibitors, might potently prevent caradiovascular remodeling associated with intermittent hypoxic stress. In this study, we evaluate the hypothesis that pitavastatin can reduce the oxidative stress and protect against LV remodeling induced by intermittent hypoxic stress in non-hyperlipidemic lean mice.

\section{METHODS}

Animals

Eight-week-old male C57BL/6J mice (purchased from Clea Japan, Osaka, Japan) were used for the study. The animals were housed under a 12:12 hour light-dark cycle, and were allowed free access to standard chow and tap water.

${ }^{1}$ Department of Internal Medicine III, Osaka Medical College, Takatsuki, Osaka, Japan and 2Laboratory of Pathological and Molecular Pharmacology, Osaka University of Pharmaceutical Sciences, Takatsuki, Osaka, Japan

Correspondence: Dr T Hayashi, Department of Internal Medicine III, Osaka Medical College, 2-7 Daigakumachi, Takatsuki, Osaka 569-8686, Japan.

E-mail: in3015@poh.osaka-med.ac.jp

Received 3 September 2009; revised 29 December 2009; accepted 3 February 2010; published online 19 March 2010 
The experimental protocol and the methods of animal care during the experiments were approved by the Experimental Animal Research Committee, Osaka University of Pharmaceutical Sciences, and all of the studies were conducted in accordance with the National Institutes of Health Guideline for the Care and Use of Laboratory Animals.

\section{Experimental protocol}

The animals were divided into two groups. One group was placed in a chamber to expose the animals to intermittent hypoxia ( $30 \mathrm{~s}$ exposure to $5 \%$ oxygen, followed by $30 \mathrm{~s}$ exposure to $21 \%$ oxygen) for $8 \mathrm{~h}$ per day during the daytime, and the other group was housed under normoxic conditions in a same room for 10 consecutive days. Each group was divided further into two groups, one treated with pitavastatin by gavage at $3 \mathrm{mg} \mathrm{kg}^{-1}$ per day and the other administered vehicle $(0.5 \%$ carboxymethyl cellulose). The dose of pitavastatin used in previous studies ranged from 0.3 to $10 \mathrm{mg} \mathrm{kg}^{-1}$ per day, and we adopted the same dose range in this study, with the expectation of the antioxidant effect of the drug in the mice. ${ }^{14-16}$

Twenty hours after the last hypoxic exposure, cardiac catheterization was performed under anesthesia induced by intraperitoneal injection of sodium pentobarbital $\left(50 \mathrm{mg} \mathrm{kg}^{-1}\right.$, i.p.), for measurement of the right ventricular systolic pressure and LV systolic pressure by a previously reported method. ${ }^{5}$ Blood collection was used to measure the plasma levels of lipid peroxide (LPO) and total cholesterol $(\mathrm{CHO})$. The heart was excised, and the upper half was subjected to light-microscopic examination. The free wall of the LV myocardium was also excised for microscopic examination, immunohistochemistry and RT-PCR.

\section{Histological examination}

Isolated ventricular tissues were fixed in $10 \%$ formaldehyde, embedded in paraffin and cut into $4 \mu \mathrm{m}$ sections. To evaluate the mean cardiomyocyte diameter, the shortest diameters of 100 cardiomyocytes in each sample were measured using NIS-Elements ver.3.07 software (Nikon, Tokyo, Japan), only in nucleated transverse sections stained with hematoxylin-eosin under a light microscope (Nikon ECLIPSE 80i) at a magnification of $\times 400$. After staining with Sirius red, the percent area of interstitial fibrosis was calculated by a previously reported method. ${ }^{17,18}$

\section{Terminal deoxynucleotidyl transferase-mediated dUTP-biotin end labeling study}

For the determination of apoptosis of cardiomyocytes in LV myocardium, a terminal deoxynucleotidyl transferase-mediated dUTP-biotin end labeling (TUNEL) study was performed. An ApopTag peroxidase in situ apoptosis detection kit (Millipore Japan, Tokyo, Japan) was used for this study according to the manufacturer's recommendations. As negative controls, distilled water was used instead of the terminal deoxynucleotidyl transferase enzyme solution, and the endometrium was used for the positive control. After counterstaining with Mayer's hematoxylin, the number of TUNEL-positive myocardial cell was counted by the method previously published. ${ }^{19}$

\section{Immunohistochemistry for 4-hydroxy-2-nonenal protein expression}

Immunohistochemical staining to determine the expression level of 4-hydroxy2-nonenal (4-HNE) was performed by a previously described method. ${ }^{5}$ Briefly, paraffin sections of the LV myocardium were incubated with a monoclonal antibody for 4-HNE (product No. MHN-20, Japan Institute for the Control of Aging, Shizuoka, Japan) and a secondary antibody (biotinylated anti-mouse IgG), followed by the addition of Vectastain Elite $\mathrm{ABC}$ reagent (Vector Laboratories, Burlingame, CA, USA). The percent area of 4-HNE staining was measured by quantitative analysis, as reported earlier. ${ }^{20}$

\section{Detection of superoxide in the LV myocardium}

To detect ROS production in situ, fresh frozen sections of the LV myocardium were stained with dihydroethidium (DHE; Molecular Probes, Eugene, OR, USA) ${ }^{21}$ Frozen sections ( $20 \mu \mathrm{m}$ in thickness) were covered with $10 \mu \mathrm{mol} \mathrm{l}^{-1}$ of
DHE solution and incubated for $30 \mathrm{~min}$ in a light-protected humidified chamber at $37^{\circ} \mathrm{C}$. Then, the sections were coverslipped and observed under a fluorescence microscope (KEYENCE BZ-8000; Osaka, Japan). ${ }^{22}$ DHE fluorescence intensity was quantified using NIH Image 1.61 software and expressed as a percentage of total cross-sectional area.

\section{Quantitative real-time RT-PCR}

Total RNA was extracted from the LV myocardial tissues using an RNeasy Mini kit, in accordance with the manufacturer instructions (Qiagen, Valencia, CA, USA). Reverse transcription was performed with random hexamers and Superscript reverse transcriptase (Invitrogen, Carlsbad, CA, USA). Real-time quantitative PCR was performed with an ABI Step One sequence detector (PE Applied Biosystems, Foster City, CA, USA). The Taqman Probe and primers for the target mRNA (Ma00443258 for TNF- $\alpha$, Ma00441724 for TGF- $\beta$ ) were purchased from Applied Biosystems. The reaction conditions were as follows: $50^{\circ} \mathrm{C}$ for $2 \mathrm{~min}, 95^{\circ} \mathrm{C}$ for $10 \mathrm{~min}$ and then 40 cycles of $95^{\circ} \mathrm{C}$ for $15 \mathrm{~s}$ and $60^{\circ} \mathrm{C}$ for $1 \mathrm{~min}$. The level of the target mRNA was normalized to that of GAPDH mRNA.

\section{Statistical analysis}

Data were presented as mean \pm s.e.m. Statistical analysis was performed using two-way ANOVA, hypoxic stress (normoxia vs. hypoxia) and treatment (pitavastatin vs. vehicle) were considered as factors including interaction, followed by Tukey-Kramer multiple comparison tests. Significance was recognized at $P<0.05$.

\section{RESULTS}

\section{Heart and body weights}

Intermittent hypoxic stress significantly decreased the body weight and tended to increase the heart weight, resulting in a significant increase in the heart weight/body weight ratio. Treatment with pitavastatin had no effect on the body weight, heart weight or the heart weight/body weight ratio (Table 1).

\section{Hemodynamic measurements}

The RVSP was increased in the mice exposed to intermittent hypoxia $(P=0.0594)$, which was not affected by the treatment with pitavastatin. There were no significant differences in the LV systolic pressure among the four groups (Table 1).

\section{LPO and CHO levels in the plasma}

The plasma LPO levels were significantly increased by intermittent hypoxia, which was significantly reduced by the treatment with pitavastatin. There were no significant differences among the four groups, although pitavastatin tended to decrease the plasma $\mathrm{CHO}$ in the mice exposed to intermittent hypoxia (Table 1). Interaction $P$-value refers to interaction by hypoxic stress and statin treatment suggested that pitavastatin was effective to lower $\mathrm{CHO}$ levels in hypoxic conditions (Table 2).

\section{Histological findings}

Intermittent hypoxia significantly increased the mean diameter of the cardiomyocytes, myofiber disarrangement and perivascular fibrosis in the LV myocardium (Figures 1 and 2). Treatment with pitavastatin significantly reduced the cardiomyocyte diameter and the severity of the perivascular fibrosis (Table 2). Furthermore, the cardiomyocyte degeneration, including disarray and scarcity of myofibril, induced by the hypoxic stress was attenuated.

\section{TUNEL study}

The number of TUNEL-positive myocardial cells significantly increased in the mice exposed to intermittent hypoxia, which was significantly suppressed by treatment with pitavastatin (Figure 3; Table 2). 
Table 1 Effect of hypoxia on the hemodynamic parameters and plasma levels of lipid peroxide (LPO) and total cholesterol (CHO)

\begin{tabular}{|c|c|c|c|c|}
\hline \multirow[b]{2}{*}{ Parameters } & \multicolumn{2}{|c|}{ Normoxia } & \multicolumn{2}{|c|}{ Hypoxia } \\
\hline & Vehicle & Statin & Vehicle & Statin \\
\hline HW (mg) & $104 \pm 3.9$ & $108 \pm 2.4(10)$ & $109 \pm 3.4(11)$ & $103 \pm 2.8(11)$ \\
\hline HW/BW $\left(\mathrm{mgg}^{-1}\right)$ & $4.3 \pm 0.1(14)$ & $4.6 \pm 0.1(10)$ & $4.9 \pm 0.2(11)^{*}$ & $4.7 \pm 0.1(11)$ \\
\hline LVSP (mm Hg) & $93.3 \pm 3.8(6)$ & $92.6 \pm 3.4(5)$ & $92.5 \pm 3.5(11)$ & $99.2 \pm 3.8(9)$ \\
\hline LPO $\left(\mathrm{nmol} \mathrm{ml^{-1 } )}\right.$ & $5.2 \pm 0.6(8)$ & $3.9 \pm 0.3(7)$ & $7.5 \pm 0.6(8)^{*}$ & $4.5 \pm 0.3(7)^{\dagger}$ \\
\hline $\mathrm{CHO}\left(\mathrm{nmol} \mathrm{ml} \mathrm{l}^{-1}\right)$ & $80 \pm 1.2(6)$ & $84 \pm 1.4(7)$ & $84 \pm 1.6(6)$ & $78 \pm 2.8(7)$ \\
\hline
\end{tabular}

Abbreviations: BW, body weight; HW, heart weight; LVSP, left ventricular systolic pressure; RVSP, right ventricular systolic pressure.

Values are means \pm s.e.m. Number of mice in parentheses.

${ }^{*} P<0.05$ vs. normoxic mice treated with vehicle.

${ }^{\dagger} P<0.05 v$ s. hypoxic mice treated with vehicle.

Table $2 P$-value of two-way ANOVA in each parameter

\begin{tabular}{lccc}
\hline & Statin & Hypoxia & $\begin{array}{c}\text { Statin } \times \text { hypoxia } \\
\text { interaction }\end{array}$ \\
\hline BW & 0.5 & 0.002 & 0.6 \\
HW & 0.7 & 0.95 & 0.2 \\
HW/BW & 0.96 & 0.018 & 0.086 \\
RVSP & 0.7 & 0.011 & 0.9 \\
LVSP & 0.5 & 0.5 & 0.4 \\
LPO & 0.0002 & 0.007 & 0.097 \\
CHO & 0.5 & 0.7 & 0.01 \\
Diameter & $<0.0001$ & $<0.0001$ & 0.012 \\
Fibrosis & 0.0002 & $<0.0001$ & $<0.0001$ \\
$4-H N E$ & 0.011 & 0.004 & 0.094 \\
DHE & $<0.0001$ & $<0.0001$ & $<0.0001$ \\
TNF- $\alpha$ & 0.0004 & 0.017 & 0.024 \\
TGF- $\beta$ & 0.0495 & 0.002 & 0.011 \\
Apoptosis & 0.0002 & $<0.0001$ & 0.0003 \\
\hline
\end{tabular}

Abbreviations: BW, body weight; $\mathrm{CHO}$, total cholesterol; $\mathrm{DHE}$, dihydroethidium labeling; diameter, mean diameter of cardiomyocytes; fibrosis, percent area of interstitial fibrosis; 4-HNE, 4-hydroxy-2-nonenal protein expression; HW, heart weight; hypoxia, exposure to hypoxia or kept in normoxia; LPO, lipid peroxide; LVSP, left ventricular systolic pressure; statin, treatment with statin or vehicle; RVSP, right ventricular systolic pressure.

\section{Immunohistochemistry for 4-HNE and DHE labeling}

Expression of 4-HNE protein, a specific product of lipid peroxidation and a marker of oxidative stress, was significantly increased in the LV myocardium of the mice exposed to intermittent hypoxia (Figure 4). DHE staining of the LV myocardium was also significantly increased in the mice exposed to intermittent hypoxia, suggesting increased superoxide production (Figure 5). Treatment with pitavastatin led to a significant decrease in the expression level of the 4-HNE protein and DHE staining in the LV myocardium. There was interaction between pitavastatin treatment and hypoxic condition in the influences on superoxide production (Table 2).

\section{Quantitative real-time RT-PCR}

Intermittent hypoxic stress significantly increased the mRNA expression levels of TNF- $\alpha$ and TGF- $\beta$ in the LV myocardium, which were significantly reduced by treatment with pitavastatin (Figure 6; Table 2).

\section{DISCUSSION}

We have reported that pitavastatin reduces oxidative stress, superoxide production and inflammatory cytokine expression in the LV myocardium, consequently reducing TUNEL-positive myocardial cells and attenuating intermittent hypoxia-induced histological changes in lean mice, independent of the LV pressure.

The existence of a correlation has been reported between the severity of sleep apnea and the incidence of hypertension. ${ }^{23}$ In this study, intermittent hypoxia for 10 days caused LV remodeling, namely, hypertrophy of the cardiomyocytes and perivascular fibrosis in the LV myocardium, without affecting the LV systolic pressure. We have already indicated that elevated RV pressure may have little effect on the LV myocardium. ${ }^{24}$ Therefore, the LV remodeling observed in this study might be caused by the intermittent hypoxic stress itself. However, Dematteis et al. ${ }^{25}$ showed that exposure to intermittent hypoxia for 35 days was associated with hypertensive surges during exposure to hypoxic conditions. Monitoring of the blood pressure during exposure to hypoxia and observation for a longer period are, therefore, necessary to elucidate the pathophysiology of intermittent hypoxia-induced cardiovascular remodeling.

Recent studies have shown significant increase in the oxidative stress in patients with obstructive sleep apnea syndrome as compared with that in unaffected subjects. ${ }^{26}$ In our experiment, intermittent hypoxia increased the plasma levels of LPO, expression levels of 4-HNE and DHE fluorescence in the LV myocardium. The increase in intercellular oxidative stress and ROS production may be attributable to the sudden increase in the oxygen tension following re-oxygenation after cellular adaptation to a low-oxygen environment during exposure to hypoxia. ${ }^{27}$ Cardiomyocytes, especially the cellular mitochondria, are considered to have an oxygen-sensing function and generate ROS after exposure to hypoxia, which might cause cardiac hypertrophy and heart failure. ${ }^{28}$ In this study, intermittent hypoxia significantly increased the number of TUNEL-positive myocardial cells, suggesting that apoptosis might be stimulated by intermittent hypoxic stress. It has been reported that pro-apoptotic proteins are upregulated and antiapoptotic proteins are downregulated in chronic sustained hypoxia. ${ }^{29}$ Furthermore, intermittent hypoxia has been shown to induce an earlier and more extensive apoptotic response of rat pheochromocytoma PC-12 cells than sustained hypoxia. ${ }^{30}$ Intriguingly, Yoshida et al. ${ }^{31}$ reported that pitavastatin was effective to suppress the oxidative DNA-damage-ATM-p53-apoptosis pathway 
a
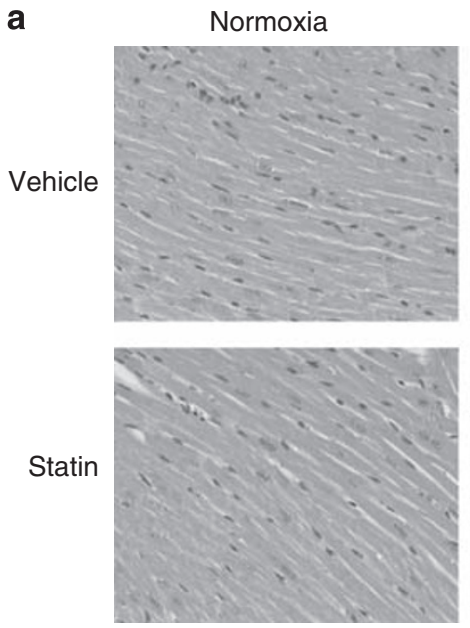
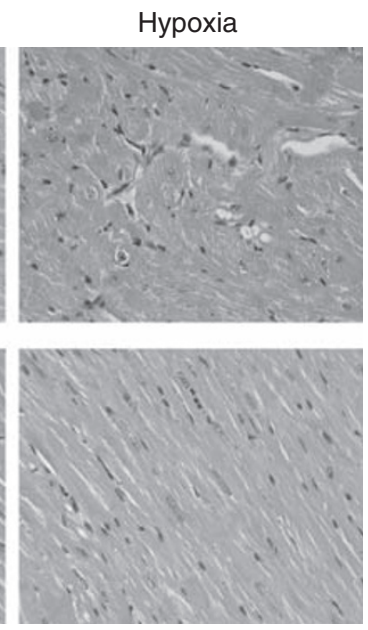

b
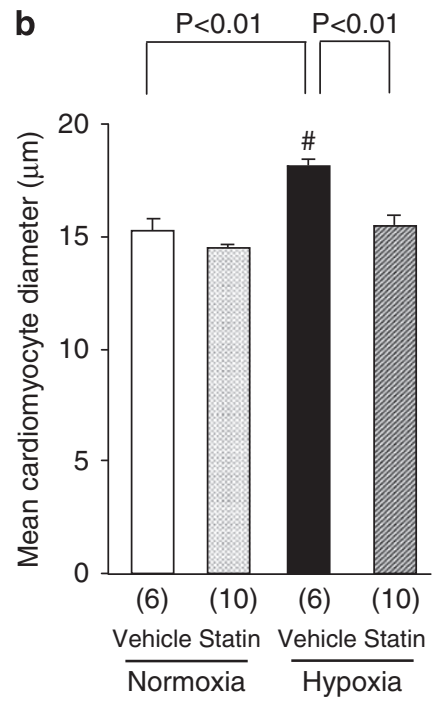

Figure 1 (a) Representative light micrographs of the LV myocardium. Intermittent hypoxia caused cardiomyocyte hypertrophy, myofiber disarrangement and nuclear degeneration. Hematoxylin-eosin stain; original magnification $\times 200$. (b) Effect of hypoxic stress on the mean diameter of the cardiomyocytes in the LV myocardium. The mean diameter of the cardiomyocytes was significantly increased in the mice exposed to intermittent hypoxia. Treatment with pitavastatin (statin) significantly suppressed the cardiomyocyte diameter and prevented the cardiomyocyte degeneration induced by hypoxic stress. Values are mean \pm s.e.m. of number of animals in parentheses. ${ }^{\#} P<0.01$ vs. normoxic mice treated with statin.

a

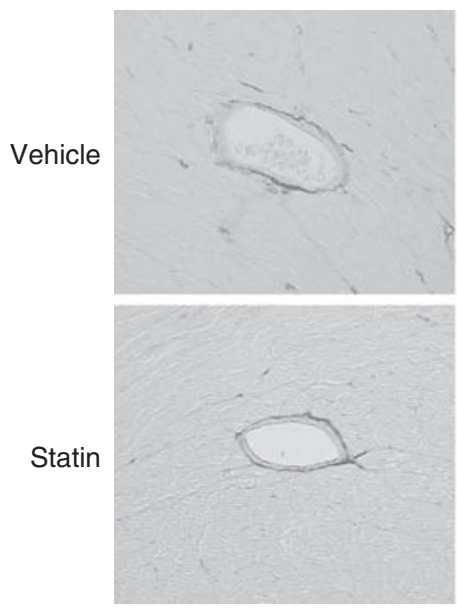

Hypoxia

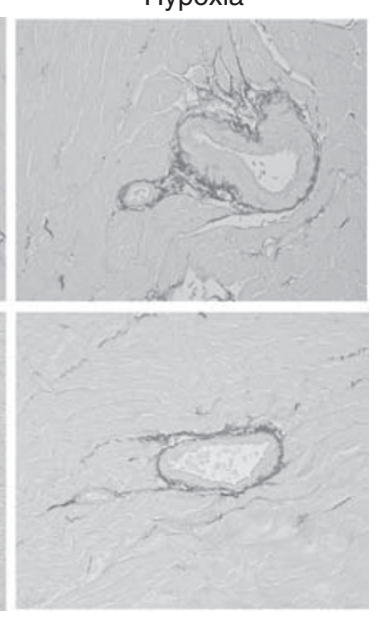

b

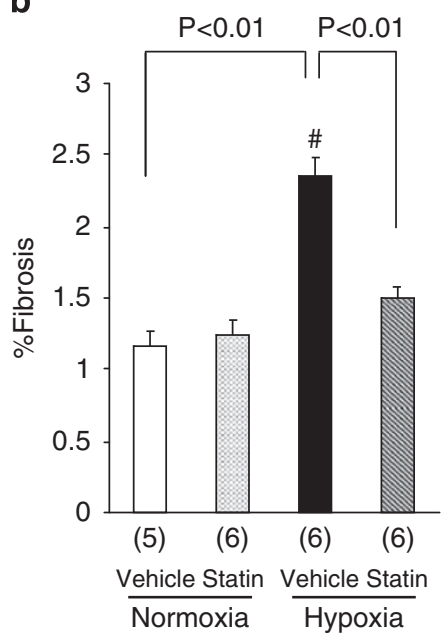

Figure 2 (a) Representative light micrographs of the LV myocardium. Sirius red stain; original magnification $\times 200$. (b) Effect of hypoxic stress on the collagen volume fraction (percentage of perivascular fibrosis) in the LV myocardium. Perivascular fibrosis was significantly increased in the mice exposed to intermittent hypoxia, which was significantly suppressed by treatment with pitavastatin. Values are mean \pm s.e.m. of number of animals in parentheses. ${ }^{\#} P<0.01$ vs. normoxic mice treated with statin.

through the inhibition of Racl activity. Although we have not confirmed apoptotic bodies in the LV myocardium, further studies focused on apoptosis induced by intermittent hypoxia should be addressed in the near future.

On the other hand, NADPH oxidase has been thought as an alternative molecule capable of $\mathrm{O}_{2}$ sensing, and ROS have been proposed to have a role in signaling the downstream effector molecules during the acute changes in response to altered oxygen tension. ${ }^{32}$ Furthermore, NADPH oxidase activity has been shown to be associated with cardiac hypertrophy and the pathogenesis of heart failure. ${ }^{33,34}$ We have shown that hypoxia-induced histological degeneration was prevented in $\mathrm{gp} 91^{-/-}$mice, suggesting that $\mathrm{gp} 91^{\text {phox }_{-} \text {containing }}$
NADPH oxidase has a crucial role in the pathophysiology of intermittent hypoxia-induced LV remodeling associated with increase in oxidative stress. ${ }^{5}$ Pitavastatin significantly reduced the plasma levels of LPO, which were increased by exposure to intermittent hypoxia, without affecting the plasma cholesterol level. Furthermore, the expression levels of 4-HNE and superoxide production in the LV myocardium were also significantly suppressed. These data suggest that pitavastatin is efficacious against oxidative stress and exerts an antioxidative effect in tissues showing high levels of oxygen consumption. We did not evaluate other tissues such as the brain, kidney or liver, and further analysis is needed to show the tissue-selective effects of pitavastatin. 
a

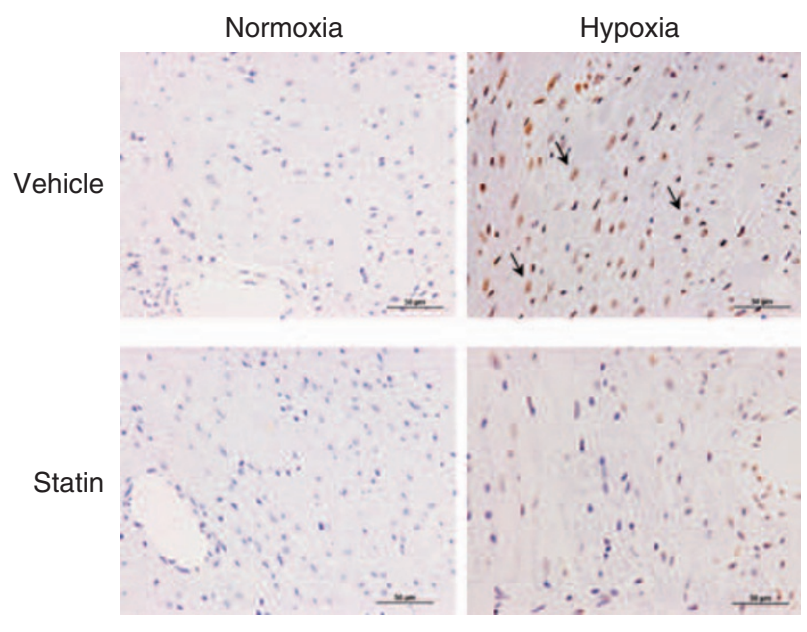

b

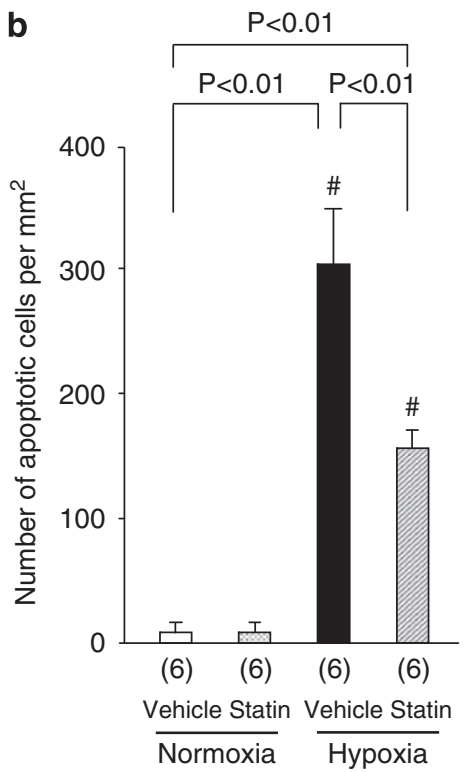

Figure 3 Terminal deoxynucleotidyl transferase-mediated dUTP-biotin end labeling (TUNEL) study. (a) TUNEL-positive myocardial cells (arrows) as well as interstitial cells were increased in mice exposed to intermittent hypoxia. (b) The number of TUNEL-positive myocardial cells significantly increased in the hypoxic mice, which was significantly suppressed by treatment with pitavastatin. Values are mean \pm s.e.m. of number of animals in parentheses. $\# P<0.01$ vs. normoxic mice treated with statin.
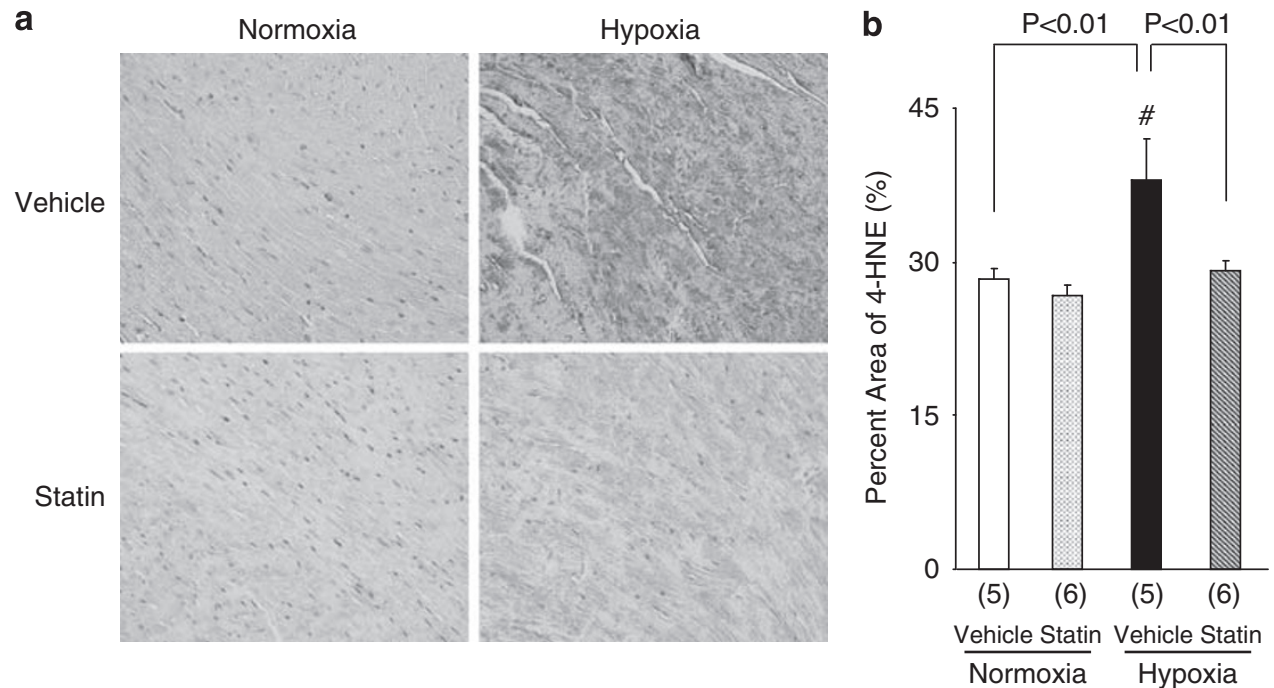

Figure 4 (a) Immunohistochemistry for 4-HNE expression in the LV myocardium. (b) Effect of hypoxic stress on the expression of 4-HNE in the LV myocardium. 4-HNE expression was significantly increased in the mice exposed to intermittent hypoxia, which was restored to normal after treatment with pitavastatin. Values are mean \pm s.e.m. of number of animals in parentheses. ${ }^{\#} P<0.01$ vs. normoxic mice treated with statin.

We have reported that treatment with an angiotensin II type 1 receptor blocker prevented hypoxia-induced LV remodeling in mice and that the renin-angiotensin system might be involved in the development of cardiac remodeling associated with exposure to hypoxia. ${ }^{18,24}$ Furthermore, a chymase inhibitor successfully suppressed the intermittent hypoxia-induced LV remodeling. ${ }^{35}$ The renin-angiotensin system has been reported to interact with pro-inflammatory cytokines and activate a redox-sensitive transcription factor termed nuclear factor- $\kappa \mathrm{B}(\mathrm{NF}-\kappa \mathrm{B}) .{ }^{36}$ The activation of NF- $\kappa \mathrm{B}$ by angiotensin II might be critical for the activation of nitric oxide, chemokines and cell adhesion molecules. ${ }^{37}$ Furthermore, Kalra et al. ${ }^{38}$ have reported that angiotensin II induces TNF- $\alpha$ mRNA and protein synthesis in the adult heart through an NF- $\kappa \mathrm{B}$-dependent pathway.

Recent studies have shown that the serum TNF- $\alpha$ levels are significantly increased in patients with sleep apnea and metabolic syndrome. ${ }^{39,40}$ We also reported elevated interleukin- 6 , TNF- $\alpha$ mRNA and NF- $\kappa \mathrm{B}$-binding activity in the LV myocardium of mice exposed to intermittent hypoxia., ${ }^{5,18}$ These results suggest that inflammatory cytokines might be involved in the LV remodeling induced by intermittent hypoxia. In this study, pitavaststin reduced the mRNA expression level of TNF- $\alpha$ in the LV myocardium, thereby preventing LV remodeling. Atrovastatin has been reported to inhibit NF- $\mathrm{B}$ 


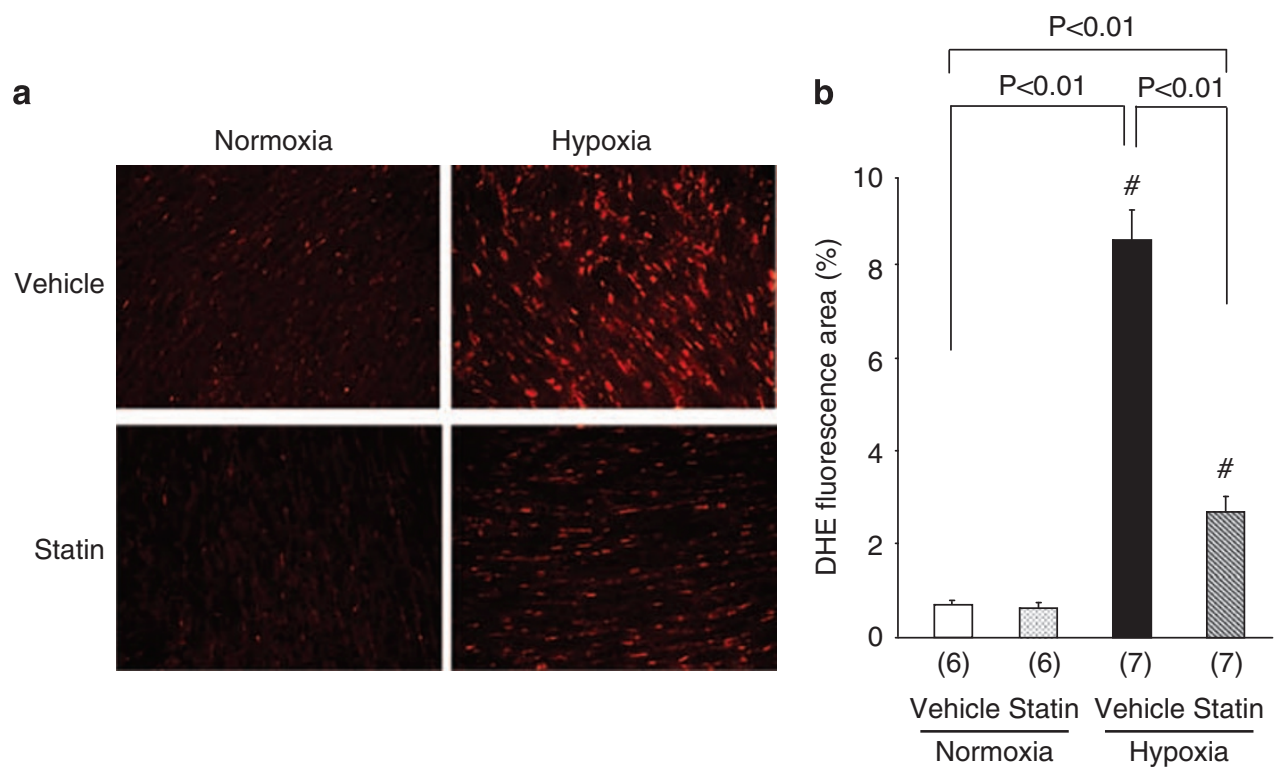

Figure 5 (a) DHE staining of the LV myocardium. (b) Effect of hypoxic stress on the expression of DHE in the LV myocardium. The intensity of DHE staining was significantly increased in the mice exposed to intermittent hypoxia, suggesting increased superoxide production. Treatment with pitavastatin led to a significant decrease in the degree of DHE staining in the LV myocardium. Values are mean \pm s.e.m. of number of animals in parentheses. ${ }^{P} P<0.01$ vs. normoxic mice treated with statin.
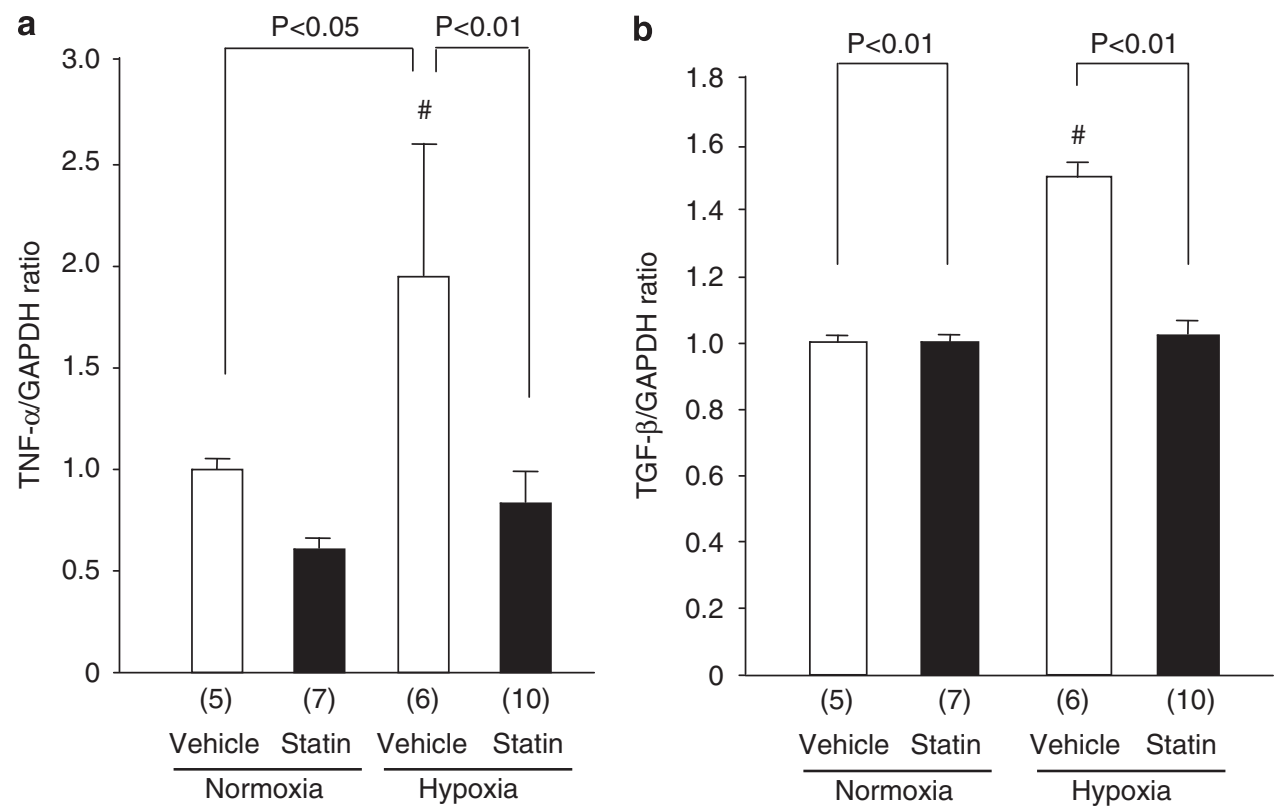

Figure 6 (a) Quantitative real-time RT-PCR for TNF- $\alpha$ (a) and TGF- $\beta$ (b) in the LV myocardium. Intermittent hypoxic stress significantly increased the mRNA expressions of TNF- $\alpha$ and TGF- $\beta$ in the LV myocardium, which were significantly reduced by treatment with pitavastatin. Values are mean \pm s.e.m. of number of animals in parentheses. ${ }^{\#} P<0.01$ vs. normoxic mice treated with statin.

activity and reduce chemokine gene expression in vascular smooth muscle cells and mononuclear cells stimulated with angiotensin II or TNF- $\alpha .{ }^{41}$ Fliser et al. ${ }^{42}$ reported that pravastatin therapy decreased the serum levels of high-sensitivity C-reactive protein, TNF- $\alpha$ and interleukin-6 in patients with essential hypertension. Taken together with our results, reduction of the plasma levels of inflammatory cytokines by statin treatment may be closely involved in the suppression of LV remodeling.
TGF- $\beta$ has a major role in the tissue fibro-inflammatory response. ${ }^{43}$ It has been reported that overexpression of TGF- $\beta$ enhanced extracellular matrix protein synthesis and led to cardiomyocyte hypertrophy. ${ }^{44}$ Yagi et al. ${ }^{16}$ reported that angiotensin II induced LV hypertrophy and oxidative stress, and that treatment with pitavastatin attenuated these changes through suppression of the TGF- $\beta /$ smads signaling pathway, although the precise mechanisms involved in the pathway are still unclear. In this study, expression of TGF- $\beta$ mRNA in 
the LV myocardium was significantly increased by exposure to intermittent hypoxia, which was suppressed by treatment with pitavastatin. Mast cells have been known to store TGF- $\beta$ in the intracellular granules and secrete it, to be activated by chymase. ${ }^{45-47}$ Further studies are needed to elucidate the role of mast cells in hypoxiainduced LV remodeling.

In conclusion, intermittent hypoxia might induce cardiovascular remodeling through oxidative stress and induction of inflammation. Pitavastatin may prevent morphological changes induced by intermittent hypoxia, at least in part, through suppression of both oxidative stress and inflammation. Aggressive treatment with pitavastatin in patients with the sleep apnea syndrome might attenuate the cardiovascular remodeling induced by the increased oxidative stress in these patients.

\section{ACKNOWLEDGEMENTS}

We express our gratitude to Dr Atsushi Takeshita, Department of Pathology, Osaka Medical College, for his critical advice in the TUNEL study. We also thank Sadao Uchida, Chieko Ota, Yumiko Ogami, Minako Ito, Motoyo Kobayashi, Yasuko Mizuoka and Yoshiro Kitaguni for their expert technical assistance.

1 Lattimore JD, Celermajer DS, Wilcox I. Obstructive sleep apnea and cardiovascular disease. J Am Coll Cardiol 2003; 41: 1429-1437.

2 Shamsuzzaman AS, Gersh BJ, Somers VK. Obstructive sleep apnea: implications for cardiac and vascular disease. JAMA 2003; 290: 1906-1914.

3 Yaggi HK, Concato J, Kernan WN, Lichtman JH, Brass LM, Mohsenin V. Obstructive sleep apnea as a risk factor for stroke and death. N Engl J Med 2005; 353: 2034-2041.

4 Kinugawa $\mathrm{S}$, Tsutsui $\mathrm{H}$, Hayashidani $\mathrm{S}$, Ide T, Suematsu N, Satoh S, Utsumi $\mathrm{H}$, Takeshita A. Treatment with dimethylthiourea prevents left ventricular remodeling and failure after experimental myocardial infarction in mice: role of oxidative stress. Circ Res 2000; 87: 392-398.

5 Hayashi T, Yamashita C, Matsumoto C, Kwak CJ, Fujii K, Hirata T, Miyamura M, Mori T, Ukimura A, Okada Y, Matsumura Y, Kitaura Y. Role of gp91phox-containing NADPH oxidase in left ventricular remodeling induced by intermittent hypoxic stress. $A m \mathrm{~J}$ Physiol Heart Circ Physiol 2008; 294: H2197-H2203.

6 Laufs U, La Fata V, Plutzky J, Liao JK. Upregulation of endothelial nitric oxide synthase by HMG CoA reductase inhibitors. Circulation 1998; 97: 1129-1135.

7 Kleemann R, Princen HM, Emeis JJ, Jukema JW, Fontijn RD, Horrevoets AJ, Kooistra T, Havekes LM. Rosuvastatin reduces atherosclerosis development beyond and independent of its plasma cholesterol-lowering effect in APOE*3-Leiden transgenic mice: evidence for antiinflammatory effects of rosuvastatin. Circulation 2003; 108: 1368-1374

8 Izumi Y, Shiota M, Kusakabe H, Hikita Y, Nakao T, Nakamura Y, Muro T, Miura K, Yoshiyama M, Iwao $\mathrm{H}$. Pravastatin accelerates ischemia-induced angiogenesis through AMP-activated protein kinase. Hypertens Res 2009; 32: 675-679.

9 Ridker PM, Cannon CP, Morrow D, Rifai N, Rose LM, McCabe CH, Pfeffer MA, Braunwald E. C-reactive protein levels and outcomes after statin therapy. N Engl J Med 2005; 352: 20-28.

10 Essig M, Nguyen G, Prie D, Escoubet B, Sraer JD, Friedlander G. 3-Hydroxy-3methylglutaryl coenzyme $A$ reductase inhibitors increase fibrinolytic activity in rat aortic endothelial cells. Role of geranylgeranylation and Rho proteins. Circ Res 1998; 83: 683-690.

11 Hayashidani S, Tsutsui H, Shiomi T, Suematsu N, Kinugawa S, Ide T, Wen J, Takeshita A. Fluvastatin, a 3-hydroxy-3-methylglutaryl coenzyme a reductase inhibitor, attenuates left ventricular remodeling and failure after experimental myocardial infarction. Circulation 2002; 105: 868-873.

12 Takemoto M, Node K, Nakagami H, Liao Y, Grimm M, Takemoto Y, Kitakaze M, Liao JK. Statins as antioxidant therapy for preventing cardiac myocyte hypertrophy. J Clin Invest 2001; 108: 1429-1437.

13 Girgis RE, Li D, Zhan X, Garcia JG, Tuder RM, Hassoun PM, Johns RA. Attenuation of chronic hypoxic pulmonary hypertension by simvastatin. Am J Physiol Heart Circ Physiol 2003; 285: H938-H945.

14 Sata M, Nishimatsu H, Osuga J, Tanaka K, Ishizaka N, Ishibashi S, Hirata Y, Nagai R. Statins augment collateral growth in response to ischemia but they do not promote cancer and atherosclerosis. Hypertension 2004; 43: 1214-1220.

15 Abe M, Matsuda M, Kobayashi H, Miyata Y, Nakayama Y, Komuro R, Fukuhara A, Shimomura I. Effects of statins on adipose tissue inflammation: their inhibitory effect on MyD88-independent IRF3/IFN-beta pathway in macrophages. Arterioscler Thromb Vasc Biol 2008; 28: 871-877.

16 Yagi S, Aihara K, Ikeda Y, Sumitomo Y, Yoshida S, Ise T, Iwase T, Ishikawa K, Azuma H, Akaike M, Matsumoto T. Pitavastatin, an HMG-CoA reductase inhibitor, exerts eNOS- independent protective actions against angiotensin II induced cardiovascular remodeling and renal insufficiency. Circ Res 2008; 102: 68-76.

17 Mori T, Chen YF, Feng JA, Hayashi T, Oparil S, Perry GJ. Volume overload results in exaggerated cardiac hypertrophy in the atrial natriuretic peptide knockout mouse. Cardiovasc Res 2004; 61: 771-779.

18 Yamashita C, Hayashi T, Mori T, Tazawa N, Kwak CJ, Nakano D, Sohmiya K, Okada Y, Kitaura Y, Matsumura Y. Angiotensin II receptor blocker reduces oxidative stress and attenuates hypoxia-induced left ventricular remodeling in apolipoprotein E-knockout mice. Hypertens Res 2007; 30: 1219-1230.

19 Takeshita A, Shibayama Y. Role of mast cells in hepatic remodeling during cholestasis and its resolution: relevance to regulation of apoptosis. Exp Toxicol Pathol 2005; 56: 273-280.

20 Okuda N, Hayashi T, Mori T, Inamoto S, Okabe M, Mieno S, Horimoto H, Kitaura Y. Nifedipine enhances the cardioprotective effect of an angiotensin-II receptor blocker in an experimental animal model of heart failure. Hypertens Res 2005; 28: 431-438.

21 Miller Jr FJ, Gutterman DD, Rios CD, Heistad DD, Davidson BL. Superoxide production in vascular smooth muscle contributes to oxidative stress and impaired relaxation in atherosclerosis. Circ Res 1998; 82: 1298-1305.

22 Nakano D, Hayashi T, Tazawa N, Yamashita C, Inamoto S, Okuda N, Mori T, Sohmiya K, Kitaura Y, Okada Y, Matsumura Y. Chronic hypoxia accelerates the progression of atherosclerosis in apolipoprotein E-knockout mice. Hypertens Res 2005; 28 : 837-845.

23 Peppard PE, Young T, Palta M, Skatrud J. Prospective study of the association between sleep-disordered breathing and hypertension. N Eng/ J Med 2000; 342: 1378-1384.

24 Inamoto S, Hayashi T, Tazawa N, Mori T, Yamashita C, Nakano D, Matsumura Y, Okuda N, Sohmiya K, Sakai A, Furuya E, Kitaura Y. Angiotensin-II receptor blocker exerts cardioprotection in diabetic rats exposed to hypoxia. Circ J 2006; 70: 787-792.

25 Dematteis M, Julien C, Guillermet C, Sturm N, Lantuejoul S, Mallaret M, Levy P, Gozal E. Intermittent hypoxia induces early functional cardiovascular remodeling in mice. $A m$ J Respir Crit Care Med 2008; 177: 227-235.

26 Christou K, Markoulis N, Moulas AN, Pastaka C, Gourgoulianis KI. Reactive oxygen metabolites (ROMs) as an index of oxidative stress in obstructive sleep apnea patients. Sleep Breath 2003; 7: 105-110.

27 Lavie L. Obstructive sleep apnoea syndrome-an oxidative stress disorder. Sleep Med Rev 2003; 7: 35-51.

28 Vanden Hoek TL, Becker LB, Shao Z, Li C, Schumacker PT. Reactive oxygen species released from mitochondria during brief hypoxia induce preconditioning in cardiomyocytes. J Biol Chem 1998; 273: 18092-18098.

29 Lee SD, Kuo WW, Lin JA, Chu YF, Wang CK, Yeh YL, Wang SG, Liu JY, Chang MH, Huang CY. Effects of long-term intermittent hypoxia on mitochondrial and Fas death receptor dependent apoptotic pathways in rat hearts. Int J Cardiol 2007; 116: 348-356.

30 Gozal E, Sachleben Jr LR, Rane MJ, Vega C, Gozal D. Mild sustained and intermittent hypoxia induce apoptosis in PC-12 cells via different mechanisms. Am J Physiol Cell Physiol 2005; 288: C535-C542.

31 Yoshida M, Shiojima I, Ikeda H, Komuro I. Chronic doxorubicin cardiotoxicity is mediated by oxidative DNA damage-ATM-p53-apoptosis pathway and attenuated by pitavastatin through the inhibition of Rac1 activity. J Mol Cell Cardiol 2009; 47: 698-705.

32 Jones RD, Hancock JT, Morice AH. NADPH oxidase: a universal oxygen sensor? Free Radic Biol Med 2000; 29: 416-424.

33 Bendall JK, Cave AC, Heymes C, Gall N, Shah AM. Pivotal role of a gp91(phox)containing NADPH oxidase in angiotensin II-induced cardiac hypertrophy in mice. Circulation 2002; 105: 293-296.

34 Li JM, Gall NP, Grieve DJ, Chen M, Shah AM. Activation of NADPH oxidase during progression of cardiac hypertrophy to failure. Hypertension 2002; 40: 477-484.

35 Matsumoto C, Hayashi T, Kitada K, Yamashita C, Miyamura M, Mori T, Ukimura A, Ohkita M, Jin D, Takai S, Miyazaki M, Okada Y, Kitaura Y, Matsumura Y. Chymase plays an important role in left ventricular remodeling induced by intermittent hypoxia in mice. Hypertension 2009; 54: 164-171.

36 Brasier AR, Jamaluddin M, Han Y, Patterson C, Runge MS. Angiotensin II induces gene transcription through cell-type-dependent effects on the nuclear factor-kappaB (NFkappaB) transcription factor. Mol Cell Biochem 2000; 212: 155-169.

37 Hernandez-Presa M, Bustos C, Ortego M, Tunon J, Renedo G, Ruiz-Ortega M, Egido J. Angiotensin-converting enzyme inhibition prevents arterial nuclear factor-kappa $B$ activation, monocyte chemoattractant protein-1 expression, and macrophage infiltration in a rabbit model of early accelerated atherosclerosis. Circulation 1997; 95 : 1532-1541.

38 Kalra D, Sivasubramanian N, Mann DL. Angiotensin II induces tumor necrosis factor biosynthesis in the adult mammalian heart through a protein kinase C-dependent pathway. Circulation 2002; 105: 2198-2205.

39 Ryan S, Taylor CT, McNicholas WT. Selective activation of inflammatory pathways by intermittent hypoxia in obstructive sleep apnea syndrome. Circulation 2005; 112: 2660-2667.

40 Minoguchi K, Tazaki T, Yokoe T, Minoguchi H, Watanabe Y, Yamamoto M, Adachi M. Elevated production of tumor necrosis factor-alpha by monocytes in patients with obstructive sleep apnea syndrome. Chest 2004; 126: 1473-1479.

41 Ortego M, Bustos C, Hernandez-Presa MA, Tunon J, Diaz C, Hernandez G, Egido J. Atorvastatin reduces NF-kappaB activation and chemokine expression in vascular smooth muscle cells and mononuclear cells. Atherosclerosis 1999; 147: 253-261.

42 Fliser D, Buchholz K, Haller H. Antiinflammatory effects of angiotensin II subtype 1 receptor blockade in hypertensive patients with microinflammation. Circulation 2004 ; 110: 1103-1107. 
43 Border WA, Noble NA. Transforming growth factor beta in tissue fibrosis. N Engl J Med 1994; 331: 1286-1292.

44 Li RK, Li G, Mickle DA, Weisel RD, Merante F, Luss H, Rao V, Christakis GT, Williams WG. Overexpression of transforming growth factor-betal and insulin-like growth factor-I in patients with idiopathic hypertrophic cardiomyopathy. Circulation 1997; 96: 874-881.

45 Pennington DW, Lopez AR, Thomas PS, Peck C, Gold WM. Dog mastocytoma cells produce transforming growth factor beta 1. J Clin Invest 1992; 90: 35-41.
46 Lindstedt KA, Wang Y, Shiota N, Saarinen J, Hyytiainen M, Kokkonen JO, Keski-Oja J, Kovanen PT. Activation of paracrine TGF-betal signaling upon stimulation and degranulation of rat serosal mast cells: a novel function for chymase. FASEB $J$ 2001; 15: 1377-1388.

47 Zhao XY, Zhao LY, Zheng QS, Su JL, Guan H, Shang FJ, Niu XL, He YP, Lu XL. Chymase induces profibrotic response via transforming growth factor-beta $1 / \mathrm{Smad}$ activation in rat cardiac fibroblasts. Mol Cell Biochem 2008; 310: 159-166. 